\title{
Review of Pharmacologic Receptors and Advances
}

\author{
Chukwu LC, Ekenjoku AJ, Okam P.C, Ohadoma SC, Olisa CL
}

\begin{abstract}
The effects of most drugs result from their interaction with macromolecular components of the same organism. These interactions have the capacity to alter the functions of the pertinent component and thereby initiate a cascade of biochemical and physiological changes that are characteristic of the response to the drug in question. The term receptor therefore, denotes the component of the organism with which the chemical agent is presumed to interact.
\end{abstract}

This drug-receptor is closely related to the enzyme-substrate complexes or that of antigen and antibody; these interactions have many common features, perhaps the most noteworthy being specificity of the receptor for a given ligand. However, the receptor not only has the ability to recognize a ligand, but can also couple or transduce this binding into a response by causing a conformational change or a biochemical effect

The concept of drugs acting on receptors generally is credited to John Langley (1878) while the word receptor was introduced in 1909 by Paul Ehrlich. A J Clark was the first to quantify drug-induced biological responses (using an equation described firstly by A V Hill in 1909 and then in 1910) and proposed a model to explain drug-mediated receptor activation. So far, nearly all of the quantitative theoretical modelling of receptor function has centred on ligand-gated ion channels and $G$ protein-coupled receptors (GPCRs). However, the richest sources of therapeutically exploitable pharmacologic receptors are proteins that are responsible for transducing extracellular signals into intracellular responses. These receptors may be divided into four families: 1) ligand-gated ion channels, 2) G protein-coupled receptors, 3) enzyme-linked receptors, and 4) intracellular receptors. The type of receptor a ligand will interact with depends on the nature of the ligand. Hydrophobic ligands interact with receptors located on the cell surface (families 1, 2, and 3). In contrast, hydrophobic ligands can enter cells through the lipid bilayers of the cell membrane to interact with receptors found inside cells (family 4). This review also addressed the basic theoretical concepts of full and partial agonism, neutral antagonism, inverse agonism and protean and ligand-selective agonism, and the relevance of these concepts in current rational drug therapy. In this review, the relative shortage of literature in this area of study constitutes a serious knowledge gap waiting to be filled up. Hence, this very review.

Index Terms - macromolecular, biochemical and physiological changes, drug-receptor, enzyme-substrate complexes, antigen and antibody, ligands, $G$ protein-coupled receptors (GPCRs), ligand-gated ion channels, enzyme-linked receptors, intracellular receptors.

\section{INTRODUCTION TO RECEPTOR THEORIES AND RECENT ADVANCES}

Receptors are specialized proteins located on the cell

Chukwu LC, College of Medicine; Chukwuemeka Odumegwu Ojukwu University, Amaku Awka Campus Nigeria.

Ekenjoku AJ, Dept. of Pharmacology, College of Medicine; Abia State University, Aba Campus, Nigeria.

Okam P.C, Dept. of Pharmacology, College of Medicine; Nnamdi Azikiwe University, Nnnewi Campus. Nnewi Nigeria

Ohadoma SC, Dept. of Pharmacology, College of Medical Sciences; University of Calabar, Nigeria

Olisa CL Dept. of Pharmacology, College of Medicine; Nnamdi Azikiwe University, Nnnewi Campus. Nnewi Nigeria membrane as well as the inside of the cell. They can that can bind to a ligand and get triggered to alter their shape or activity giving rise to a signal cascade of intracellular molecules - the second messengers, which, ultimately, results in some change in the cell's function (Tanner Marshall et al, 2020).

Receptors are specialized target macromolecules present on the cell surface or intracellularly. Receptors bind drugs and initiate events leading to alterations in biochemical and/or biophysical activity of a cell, and consequently, the function of an organ. Drugs may interact with receptors in many different ways and may exert their effects. These possible exerted effects may be both beneficial and or harmful and are triggered by interacting with receptors. Drugs may bind to enzymes (for example, inhibition of dihydrofolate reductase by trimethoprim), nucleic acids (for example, blockade of transcription by dactinomycin), or membrane receptors (for example, alteration of membrane permeability by pilocarpine). In each case, the formation of the drug-receptor complex leads to a biologic response (Michelle, et al 2012).

Receptor theory is the application of receptor models to explain drug behaviour (Kenakin, 2008). Pharmacological receptor models preceded accurate knowledge of receptors by many years (Kenakin, 2004). John Newport Langley and Paul Ehrlich introduced the concept of a receptor that would mediate drug action at the beginning of the 20th century. A J Clark was the first to quantify drug-induced biological responses (using an equation described firstly by A V Hill in 1909 and then in 1910) and proposed a model to explain drug-mediated receptor activation. So far, nearly all of the quantitative theoretical modelling of receptor function has centred on ligand-gated ion channels and GPCRs (Rang, 2006).

The binding of drugs to receptors can involve all known types of interactions ionic, hydrogen bonding, hydrophobic, van der Waals, and covalent. Most interactions between drugs and their receptors involve bonds of multiple types. If binding is covalent, the duration of drug action is frequently, but not necessarily, prolonged. Noncovalent interactions of high affinity also may be essentially irreversible (Iain, 2006).

Receptor-receptor interactions discovery (RRI) has expanded our understanding of the role that $G$ protein-coupled receptors (GPCRs) play in intercellular communication. The finding that GPCRs can operate as receptor complexes, and not only as monomers, suggests that several different incoming signals could already be integrated at the plasma membrane level via direct allosteric interactions between the protomers that form the complex. Most research in this field has focused on neuronal populations and has led to the identification of a large number of RRI (Diego et al, 2019).

Drug molecules combine with receptors at a rate which is 
proportional to the concentration of molecules in solution and to the number of free receptors. The resulting complex breaks down at a rate proportional only to the number of complexes. Thus the rate of combination is $k_{1} A(1-y)$ and the rate of dissociation is $k_{2} y$, where $k_{1}$ and $k_{2}$ are constants, $A$ the concentration of drug, and $y$ the proportion of receptors occupied by the drug. When the two rates are equal we have,

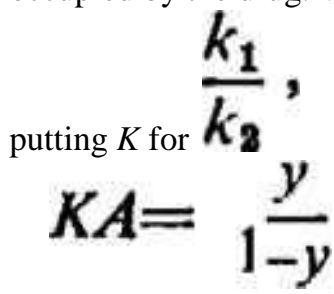

1

When $y=0.5$, then

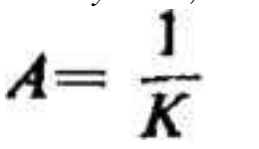

1a

Equation 1 therefore relates the concentration of drug and the proportion of receptors which are occupied by drug molecules at equilibrium. Clark, however, went a step further than this and, by taking $y$ as the response, used equation 1to relates the response of the tissue to the concentration of drug. Thus there was in Clark's treatment the implicit assumption that the percentage of receptors occupied is equal to the percentage response of the tissue. When $50 \%$ of the receptors are occupied there is $50 \%$ of the possible response; when the response is maximal all the receptors are occupied (Stephenson,

1997).

Structure-Activity Relationship and Drug Design. The strength of the reversible interaction between a drug and its receptor, as measured by their dissociation constant, is defined as the affinity of one for the other. Both the affinity of a drug for its receptor and its intrinsic activity are determined by its chemical structure. This relationship frequently is quite stringent. Relatively minor modifications in the drug molecule may result in major changes in its pharmacological properties based on altered affinity for one or more receptors (Iain, 2006).

The magnitude of the drug effect depends on the drug concentration at the receptor site, which in turn is determined by the dose of drug administered and by factors characteristic of the drug pharmacokinetic profile, such as rate of absorption, distribution, and metabolism (Michelle, et al 2012).

In considering the structural features of a molecule which determine its potency for some specific action, authors, working within the framework of Clark's ideas, have usually considered that these features exert their effects only by modifying the affinity of the molecule for the appropriate receptors. Only occasionally has some suggestion carried an implication of the concept of what has here been termed efficacy. The clear recognition of two separate entities "affinity" and "efficacy," both of which might be altered by any one structural change, should clarify the consideration of many structure-action problems (Stephenson, 1997). The stringent nature of chemical structure to specificity of binding of a drug to its receptor is illustrated by the capacity of receptors to interact selectively with optical isomers, as described for the antimuscarinic actions of L-hyoscyamine versus DL-hyoscyamine (atropine) by the classic studies of Arthur Cushney (Iain, 2006).

The importance of Isomerism in the field of clinical pharmacology and pharmacotherapeutics, can be demonstrated from the pharmacokinetic and pharmacodyamic differential properties isomers. Drug isomerism has opened a new era of drug development. Recently, the knowledge of isomerism has helped in the introduction of safer and more effective drug alternatives of the newer as well as existing drugs. Many existing drugs have undergone chiral switch i.e., switching from racemic mixture to one of its isomers. An example is Cetrizine to levocetrizine; in this an effective and safer drug has been made available. The basic concepts of stereochemistry and chirality coupled with their significance in pharmacotherapeutics has been noted Naveen et al, 2013.

Nature of Receptor-Drug interactions: Receptor occupancy model- The receptor occupancy model, which describes agonist and competitive antagonists, was built on the work of Langley, Hill and Clark. The occupancy model was the first model put forward by Clark to explain the activity of drugs at receptors and quantified the relationship between drug concentration and observed effect. It is based on mass-action kinetics and attempts to link the action of a drug to the proportion of receptors occupied by that drug at equilibrium (Christopoulos \& El-Fakahany, 1999).

Drugs for Seven Transmembrane Receptors (7TMRs) can have different behaviors in different organs. Pharmacology describes drug behavior in organ systems with system-independent scales (affinity, efficacy) and these can be used to convert descriptive data (what we see in experiments) to predictive data (what the drug will do in therapeutic systems). This conversion is essential in the industrial and academic drug discovery process. The paper views applies mathematical models of efficacy and receptor allosteric function to characterize drug activity for 7TMRs. Specifically this has produced methods to quantify agonism, biased signaling, allosteric functional effects and quantification of the effects of mutation on receptor function (Kenakin, 2008).

The G- protein coupled receptors are also known as sevenpass transmembrane receptors. This means that they are really long proteins that have one end that sits outside the cell, and then the snake- like protein dips in and out of the cell membrane seven times, and finally ends on the inside of the cell. While the ligand binds to the end sitting outside the cell, the end of the protein that is within the cell activates guanine nucleotide-binding proteins or $\mathrm{G}$ proteins, which contain an alpha, beta, and gamma subunit (Tanner Marshall et al, 2020).

In particular, the magnitude of the response is directly proportional to the amount of drug bound, and the maximum response would be elicited once all receptors were occupied at equilibrium. He applied mathematical approaches used in enzyme kinetics systematically to the effects of chemicals on tissues (Kenakin, 2004).

\section{Competitive inhibition models}

The development of the classic theory of drug antagonism by Gaddum, Schild and Arunlakshana built on the work of Langley, Hill and Clark (Coloquhoun, 1973). 
Gaddum described a model for the competitive binding of two ligands to the same receptor in short communication to the Physiological Society in 1937. The description referred only to binding, it was not immediately useful for the analysis of experimental measurements of the effects of antagonists on the response to agonists (Coloquhoun, 2006).

It was Heinz Otto Schild who made measurement of the equilibrium constant for the binding of an antagonist possible. He developed the Schild equation to determine a dose ratio, a measure of the potency of a drug. In Schild regression, the change in the dose ratio, the ratio of the EC50 of an agonist alone compared to the EC50 in the presence of a competitive antagonist as determined on a dose response curve used to determine the affinity of an antagonist for its receptor (Coloquhoun, 1973 \& Coloquhoun, 2006).

Agonist models: The flaw in Clark's receptor-occupancy model was that it was insufficient to explain the concept of partial agonist lead to the development of agonist models of drug action by Ariens in 1954 and by Stephenson in 1956 to account for the intrinsic activity (efficacy) of a drug that is, its ability to induce an effect after binding (Coloquhoun, 1973 \& Machle, et al 2002).

\section{What is Docking?}

Docking is a method which predicts the preferred orientation of one molecule to another molecule when they are bound together to form a stable complex. Molecular docking can be referred to as "lock and key" model. Here the protein can be called as lock and the ligand can be called as key. It describes the best fit orientation of the ligand required for binding to a particular protein. To perform a docking, first one may require a protein molecule. The protein structure and ligands are the inputs for docking (Amrita Lab. 2014).

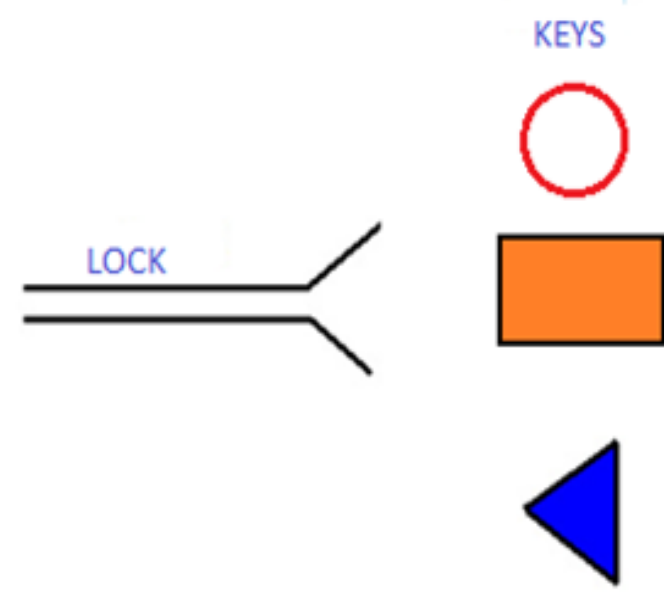

Amrita Virtual Lab. Collaborative Platform. (20014). [Ver. 00.3.2]

During drug development process, large numbers of molecules are failing at later stages due to safety or efficacy concerns which are generally investigated by conducting various experiments on animals but unfortunately, animal experimentation is associated with lots of limitations. Owing to this, a number of alternative approaches requiring less number of animals have been developed. Docking is an alternative approach for screening of compounds before actual testing on animals. Docking is the best option to predict the energetically favourable binding conformations of ligands in the active site cavity of particular receptor and has gained popularity in the scientific community to save time and money involved in the drug development process. The use of such techniques is increasing day by day among researchers due to availability of user friendly software's. But there is a lack of knowledge and confidence among scientists regarding accuracy of these software's (Meenakshi et al, 2018).

Recently Aaftaab and colleagues, (2019) noted that the process of hunt of a lead molecule is a long and a tedious and one is often demoralized by the endless possibilities one has to search through. Fortunately, computational tools have come to the rescue and have undoubtedly played a pivotal role in rationalizing the path to drug discovery. Of all techniques, molecular docking has played a crucial role in computer aided drug design and has swiftly gained ranks to secure a valuable position in the modern scenario of structure-based drug design (Aaftaab et al, 2019).

However, there are many concerns to be taken care of while carrying out any docking study. Such include the selection of correct binding sites of the target protein, the selection of correct docking pose, lack of clarity over whether the compound is an antagonist or agonist, difficult to get best matching algorithms and scoring schemes. Many compounds show high dock score, but unfortunately fail in pre-clinical studies. It is necessary to understand the method's merits, demerits, the scope of application, and interpretation so as to ensure correct and effective application of docking techniques (Aaftaab et al, 2019; Meenakshi et al, 2018).

Chemistry of Receptors and Ligands: Interaction of receptors with ligands involves the formation of chemical bonds, most commonly electrostatic and hydrogen bonds, as well as weak interactions involving van der Waals forces. These bonds are important in determining the selectivity of receptors, because the strength of these noncovalent bonds is related inversely to the distance between the interacting atoms. Therefore, the successful binding of a drug requires an exact fit of the ligand atoms with the complementary receptor atoms. The bonds are usually reversible, except for a handful of drugs (for example, the nonselective $\alpha$-receptor blocker phenoxybenzamine, and acetyl cholinesterase inhibitors in the organophosphate class) that covalently bond to their targets (Michelle et al, 2012).

\section{MAJOR RECEPTOR FAMILIES:}

Pharmacology defines a receptor as any biologic molecule to which a drug binds and produces a measurable response. Thus, enzymes and structural proteins can be considered to be pharmacologic receptors. However, the richest sources of therapeutically exploitable pharmacologic receptors are proteins that are responsible for transducing extracellular signals into intracellular responses (Michelle, 2012 \& Iain, 2006).

These receptors may be divided into four families:

I) Ligand-gated ion channels,

II) G protein-coupled receptors (Juan et al, 2016),

III) Enzyme-linked receptors, and

IV) Intracellular receptors.

The type of receptor a ligand will interact with depends on the nature of the ligand. Hydrophobic ligands interact with 
receptors that are found on the cell surface (families 1, 2, and 3 ). In contrast, hydrophobic ligands can enter cells through the lipid bilayers of the cell membrane to interact with receptors found inside cells (family 4) see above.

\section{A. LIGAND GATED ION CHANNEL}

The majority of ion channels fall into two broad categories: The Voltage Gated Ion Channel (VGIC) and

The Ligand Gated Ion Channel.

The LGIC superfamily of receptors contains an intrinsic ion channel, which undergoes a conformational change following the binding of an extracellular ligand to its site on the channel (Barnard, 1992).

Ligand-gated ion channels are transmembrane protein complexes that conduct ion flow through a channel pore in response to the binding of a neurotransmitter. They are different from voltage-gated ion channels, which are sensitive to membrane potentials, and GPCRs, which use second messengers (www.sciencedirect.com>topics.ligand-gated-ion-channel).

This conformational change allows for the opening of the ion pore, although the channel may open and close several times while the ligand is attached. This gating will cease when the ligand disassociates from the binding site (Ashcroft, 2000).

The LGIC group includes:

a. the nicotinic acetylcholine receptor ( $\mathrm{AAChR})$,

Antibodies to Nicotinic Acetylcholine Receptor

Nicotinic Acetylcholine Receptor Modulators

b. the $\gamma$-aminobutyric acid receptors $\mathrm{GABA}_{\mathrm{A}}$ and $\mathrm{GABA}_{\mathrm{C}}$,

Antibodies to GABA and Glycine Receptors

GABA and Glycine Modulators

c. the glycine receptor (GlyR), ionotropic glutamate receptors (iGluR)

Antibodies to Ionotropic Glutamate Receptors Ionotropic Glutamate Receptor Modulators and

d. The $\mathrm{P} 2 \mathrm{X}$ receptors.

$$
\begin{array}{llll}
\text { Antibodies to P2X } & \text { Receptors }
\end{array}
$$

P2X Receptor Modulators.

The ligand-gated ion channels are responsible for regulation of the flow of ions across cell membranes. The activity of these channels is regulated by the binding of a ligand to the channel. Response to these receptors is very rapid, having durations of a few milliseconds. Their functions are modified by numerous drugs. Stimulation of the nicotinic receptor by acetylcholine results in sodium influx, generation of an action potential, and activation of contraction in skeletal muscle. Benzodiazepines, on the other hand, enhance the stimulation of the GABA receptor by GABA, resulting in increased chloride influx and hyperpolarization of the respective cell. Although not ligand-gated, ion channels, such as the voltage-gated sodium channel, are important drug receptors for several drug classes, including local anesthetics (Michelle, 2012).

Ion channel receptors are a vital component of nervous system signaling. They allow rapid and direct conversion of a chemical neurotransmitter message to an electrical current. In recent years, it has become apparent that ionotropic receptors are regulated by protein-protein interactions with other ion channels, G-protein coupled receptors and intracellular proteins. These other proteins can also be modulated by these interactions with ion channel receptors. This bidirectional functional cross-talk is important for critical cellular functions such as excitotoxicity in pathological and disease states like stroke, and for the basic dynamics of activity-dependent synaptic plasticity (Shupeng et al, 2014).

\section{B. G PROTEIN-COUPLED RECEPTORS:}

G-protein coupled receptors (GPCRs): are the largest group of plasma membrane receptors of which rhodopsin and adrenergic receptors are the most familiar. They are integral plasma membrane proteins that transduce signals from extracellular ligands to signals in intracellular relay proteins, the heterotrimeric GTP binding proteins ( $\mathrm{G}$ proteins). By coupling to many downstream effectors, the $G$ proteins initiate pleiotropic changes in many targets. Thus, the extracellular signal is typically amplified to produce robust, varied, and cell-specific responses (Juan et al, 2016; Angers, et al 2002 ; Bertil, 2009).

They are receptors are involved in responses of cells to many different kinds of signals, from epinephrine, to odors, to light. In fact, a variety of physiological phenomena including vision, taste, smell and the fight-or-flight response are mediated by GPCRs. Being cell surface receptors, G-protein coupled receptors pass on the signals that they receive with the help of guanine nucleotide binding proteins (a.k.a. G-proteins). Though there are hundreds of different G-protein coupled receptors, they all have the same basic structure. All of them consist of a single polypeptide chain that threads back and forth seven times through the lipid bilayer of the plasma membrane. For this reason, they are sometimes called seven- pass transmembrane (7TM) receptors. One end of the polypeptide forms the extracellular domain that binds the signal while the other end is in the cytosol of the cell (bio.libretexts.org>...>8: signaling 2019. 8.4: Gprotein Coupled Receptorss (GPCR) Bio Libre Texts).

GPCRs are quite distinct from growth-factor receptors (e.g. insulin, NGF, BDNF etc), which signal through tyrosine kinases, do not use heterotrimeric $\mathrm{G}$ proteins, and typically regulate gene expression. However, GPCRs and growth-factor receptors do share several common final enzymatic pathways of signaling (Bertil, 2002).

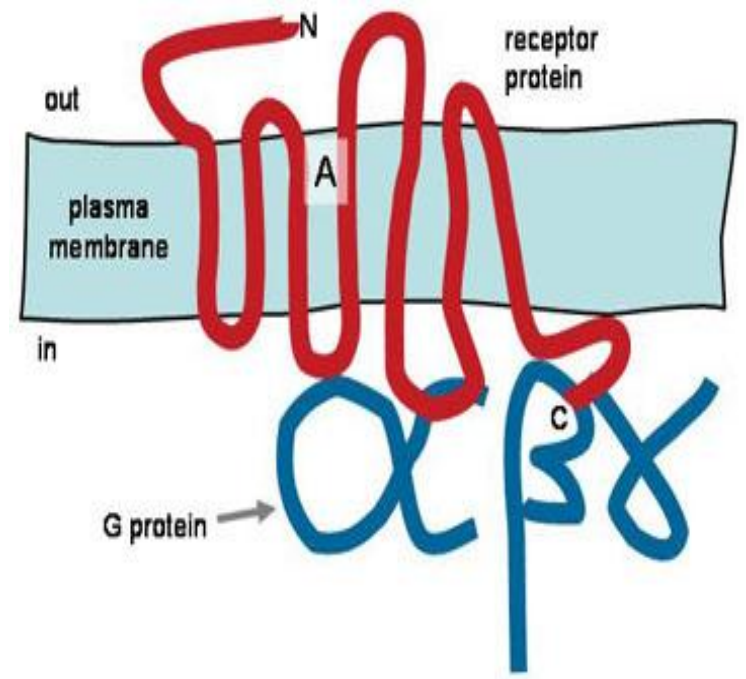

Fig: A conceptual cartoon of a G-protein coupled receptor in the plasma membrane with the characteristic seven 
$\alpha$-helical transmembrane segments.

The coupled heterotrimeric G-protein is represented schematically by the letters $\alpha, \beta, \gamma$ in the cytoplasm. Not to scale since the mass of G-protein is larger than that of the receptor

Labels: A, agonist in its binding pocket; $\mathrm{C}$, receptor C-terminus in cytoplasm; N, receptor extracellular N-terminus.

All GPCRs have a membrane topology with seven $\alpha$-helical transmembrane segments, $\mathrm{N}$-terminus outside and
C-terminus inside. Many of them have a lipid modification, palmitoylation near the C-terminus that anchors them further in the membrane. X-ray crystal structures, available so far for several dozen GPCRs, show that the transmembrane segments are broken helices that cross the membrane at various angles. With this many structures, common structural features can be deduced (Venkatakrishnan et al, 2013).

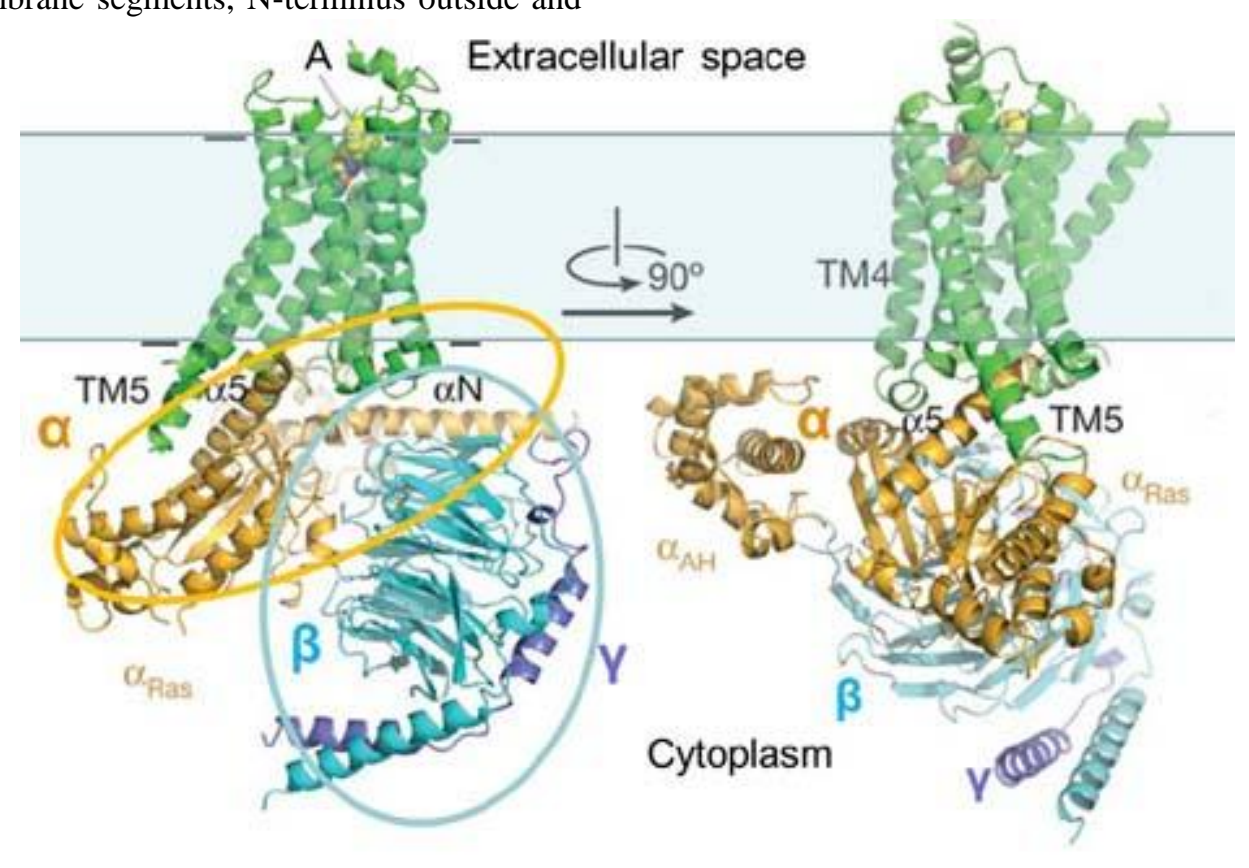

Fig: Two views of the $\beta 2$ receptor (green) activated by and enveloping an agonist (colors in upper part) and bound to the heterotrimeric $\alpha \beta \gamma \mathrm{G}$ protein Gs below on the cytoplasmic side. (Crystal structure adapted from Rasmussen et al., 2011).

Downstream coupling of Ga-GTP: There are five canonical signaling pathways

The downstream effects of stimulating a GPCR depend on which $G$ protein type(s) it couples to. Heterotrimeric G proteins are named by the type of $\alpha$ subunit they contain, and there are nearly 20 genes encoding G $\alpha$ subunits. However, for understanding much of their signaling it suffices to consider five broad $G \alpha$ families and five signaling pathways that they most typically activate:

1. $\mathbf{G} \boldsymbol{\alpha}_{\mathbf{s}}, G \boldsymbol{\alpha}_{\text {olf }}$ : the first discovered activates plasma membrane adenylyl cyclases, increasing cellular cyclic AMP (cAMP), which e.g. stimulates phosphorylation of target proteins by cAMP-dependent protein kinase. $\mathrm{G} \alpha_{\mathrm{s}}$ and its downstream signaling can be covalently activated by cholera toxin.

2. $\mathbf{G} \boldsymbol{\alpha}_{\mathbf{i}}, G \alpha_{o}$ : inhibit most adenylyl cyclases, decreasing cellular cAMP. $\mathrm{G} \alpha_{\mathrm{i}}$ and $\mathrm{G} \alpha_{\mathrm{o}}$ can be covalently inactivated and their signaling turned off by Pertussis toxin. $\mathrm{G} \alpha_{\mathrm{o}}$ is said to constitute $1 \%$ of brain proteins. Being so abundant, the PTX-sensitive $G$ proteins are also the principal source of active $\mathrm{G} \beta \gamma$ subunits.

3. $\mathbf{G} \boldsymbol{\alpha}_{\mathbf{q}}, G \alpha_{11}$ : activate phospholipase $\mathrm{C} \beta$ (PLC $\left.\beta\right)$, a lipase tha cleaves the signaling phosphoinositide lipids $\left(\mathrm{PIP}_{2}\right)$ of the plasma membrane, generating several second messengers including $\mathrm{IP}_{3}$ that releases $\mathrm{Ca}^{2+}$ from intracellular stores and diacylglycerol that activates phosphorylation by protein kinase $\mathrm{C}$.

4. $\mathbf{G} \boldsymbol{\alpha}_{12}, G \alpha_{13}$ : enhance Rho kinase, change expression of some genes, and slow dephosphorylation of myosin light chain

5. $\quad \mathbf{G}_{\text {transducin }}, \mathrm{G} \boldsymbol{\alpha}_{\text {gustducin }}$ : activate cyclic GMP (cGMP) phosphodiesterase (transducin) that cleaves and depletes cytoplasmic cGMP (in the retina only) or cAMP phosphodiesterase (gustducin) that cleaves and depletes cAMP [in some taste receptors] (Bertil, 2009).

\section{Termination of GPCR signaling}

Termination of signaling requires turning off activated receptors, turning off activated G-proteins, and return of second messenger levels, protein phosphorylation levels, and other changed metabolites to their original values. Consider the first two. Receptors quickly deactivate upon removal and unbinding of agonist ligand. They are also inactivated by other processes even while ligand is still present, mechanisms that prevent over-stimulation.(Gainetdinov, et al 2004).

Signaling without $\mathbf{G}$ proteins: GPCR activation can evoke signal pathways that do not require coupling to $G$ proteins. Much as activated receptor conformations are recognized by their complementary $G$ proteins (the classical route), so too they can be recognized by other signaling 
proteins including the GRKs, arrestins, JAK, Src family kinases, and PDZ-domain containing proteins (non-classical signaling). Such coupling can evoke clathrin-mediated internalization, activation of MAP (mitogen-activated protein) kinase, or stimulation of $\mathrm{Na}^{+} / \mathrm{H}^{+}$exchange (Sun et al., 2007).

\section{A time line:}

1. 1878-1905 Langley postulates receptor concept for action of muscarine

2. 1900-1950 Pharmacology of agonists and antagonists distinguishes many receptor subtypes phenomenologically

3. 1950-1965 Concept of the first second messenger (cAMP) formulated by Sutherland as an intermediate in adrenaline and glucagon action (Nobel Prize)

4. 1970-1980 Rodbell and Gilman recognize that a GTP binding protein conveys the receptor signal to adenylyl cyclase (Nobel Prize)

5. 1986-1990 Initial cloning reveals that many pharmacological receptors are heptahelical like rhodopsin, and the common features of coupling to G-proteins inspire the concept of GPCRs as a receptor class with a uniform paradigm of signaling. Rhodopsin is considered the prototype member of this class.

6. 2000-2013 Crystal structures of >20 GPCRs are published (Nobel Prize, Kobilka and Lefkowitz) Bertil, 2009.

\section{A. ENZYME-LINKED RECEPTORS:}

Enzyme-linked receptor proteins either possess an in-built enzyme or associate with separate enzymes in the cytoplasm. These enzymes are activated upon ligand binding. They relay the extracellular signal to the nucleus by a sequence of interactions that eventually turn on specific transcription factors, altering gene expression in the cell.

Basic receptor structure: All enzyme-linked receptors share a few common features;

\section{Ligand-binding domain}

- Extracellular to allow easy access for ligands.

- Strong affinity for specific ligands - allows different ligands that bind to the same receptor to evoke particular cellular responses.

\section{Transmembrane domain}

- Contains a series of hydrophobic amino acids.

- Tethers the receptor to the cell membrane.

3. Cytosolic "active" enzyme domain

- Either intrinsic to the receptor or tightly bound via the cytosolic domain.

- The majority are kinases; they phosphorylate specific threonine, serine, and tyrosine amino acid residues (THR, S, TY = THIRSTY). Heyes, 2014.

\section{Enzyme-linked receptor classes}

Six classes of enzyme-linked receptors have thus far been identified:

1. Receptor tyrosine kinases phosphorylate specific tyrosines on a small set of intracellular signaling proteins.

2. Tyrosine-kinase-associated receptors associate with intracellular proteins that have tyrosine kinase activity.

3. Receptorlike tyrosine phosphatases remove phosphate groups from tyrosines of specific intracellular signaling proteins. (They are called "receptorlike" because the presumptive ligands have not yet been identified, and so their receptor function has not been directly demonstrated.)

4. Receptor serine/threonine kinases phosphorylate specific serines or threonines on associated latent generegulatory proteins.

5. Receptor guanylyl cyclases directly catalyze the production of cyclic GMP in the cytosol.

6. Histidine-kinase-associated receptors activate a "two-component" signaling pathway in which the kinase phosphorylates itself on histidine and then immediately transfers the phosphate to a secondintracellular signaling protein. (www.ncbi.nih.gov>books $>$ NBK26822).
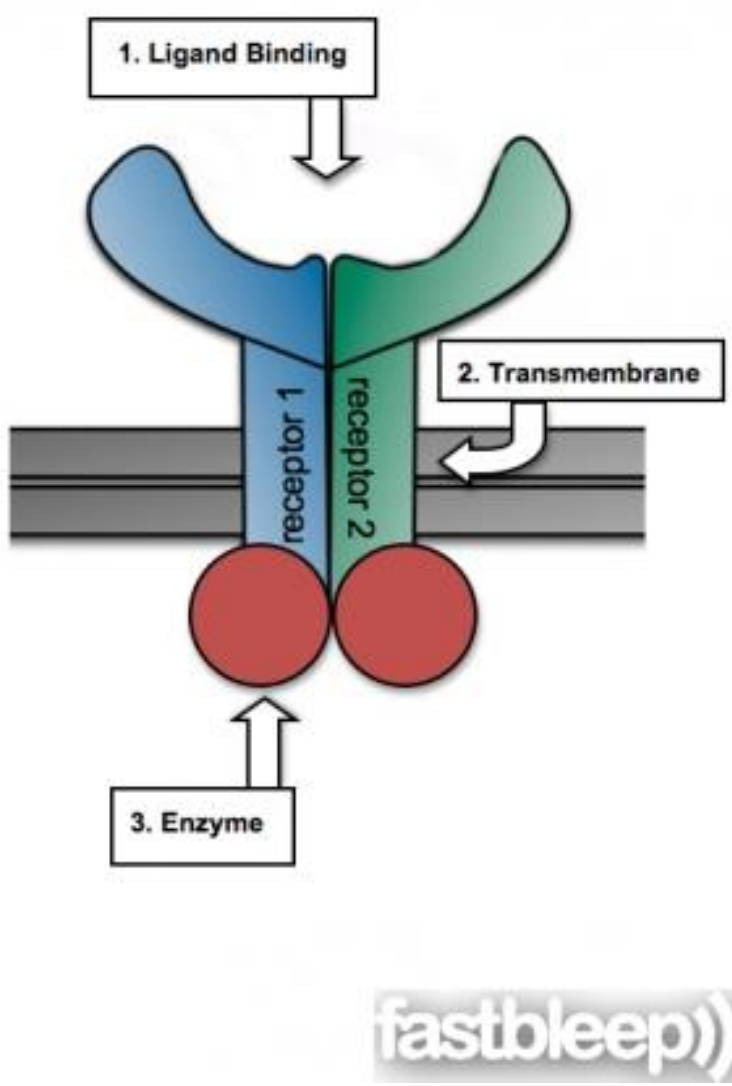

\section{Signal relay}

Within an organism, the processes of metabolism, growth, and differentiation must be tightly integrated for correct function. Cell signalling essentially connects and lubricates these occurances to ensure that progress runs smoothly.

\section{Receptor serine/threonine kinases}

There are two types of serine/threonine kinase receptors, both of which contain an intracellular kinase domain. They are each dimeric proteins, so an active receptor complex is made up of four receptors.

\section{Type I receptors}

- Inactive unless in complex with type II receptors.

- Do not interact with ligand dimers.

- Contain conserved sequences of serine and threonine residues near to their kinase domains.

\section{Type II receptors}

- Constitutively active kinase domains (even in the absence of the bound ligand). 
- Able to phosphorylate and activate the type I receptor.

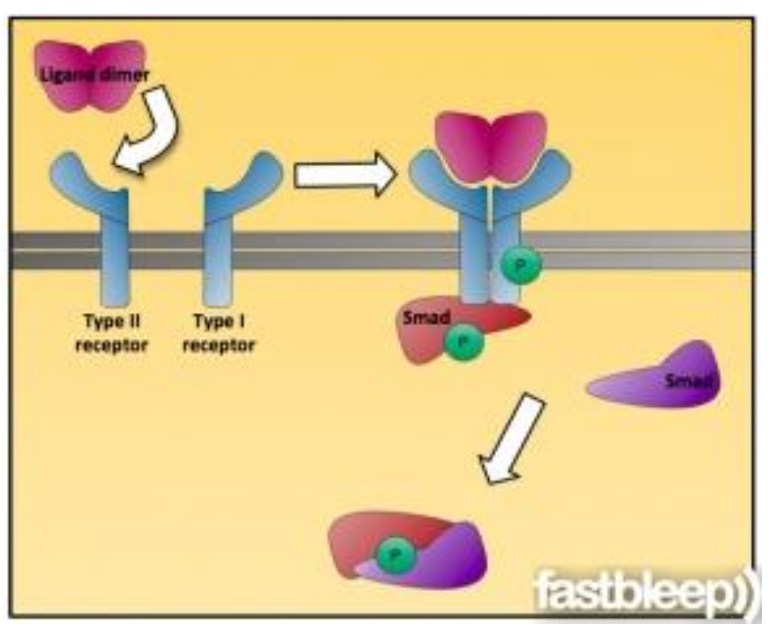

Type I receptors are kept inactive by a portion of its cytosolic domain that blocks its kinase activity.

TGFB/bone morphogenetic protein (BMP)/activin ligands bind as dimers to Type II receptors.

Type II receptors then bind to, and phosphorylate, Type I receptors. This removes the inhibition of Type I kinase activity.

Type I receptors then phosphorylate Smad transcription factors, allowing them to dimerise and enter the nucleus to repress or activate target gene expression. (http://www.youtube.com/watch?v=QoJBIfM0bmU).

\section{Receptor tyrosine kinases (RTKs)}

RTK ligands, such as fibroblast growth factor (FGF), epidermal growth factor (EGF), nerve growth factor (NGF) etc. bind as dimers.

1. Ligand binding to RTK monomers results in dimer formation.

2. Receptors possess an intracellular tyrosine kinase domain. Within the dimer the conformation is changed, locking the kinase into an active state.

3. The kinase of one receptor then phosphorylates a tyrosine residue contained in the "activation lip" of the second receptor.

This forces the activation lip out of the kinase active site, allowing ATP bind and resulting in enhanced kinase activity.

This induces phosphorylation at further tyrosine residues.

4. Phosphotyrosine is a conserved "docking site" for many intracellular signal transduction proteins that contain SH2 domains [see further down the page for more information]. (Heyes, 2014; www.ncbi.nih.gov>books >NBK26822;

www.wiley.com/college/fob/quiz/quiz21/21-15.html nd \& $\mathrm{www}$.youtube.com/watch?v=_Z1uu0rXFDc\&feature=relate d nd.

\section{B. INTRACELLULAR RECEPTORS.}

Receptors for steroid and thyroid hormones are located inside target cells, in the cytoplasm or nucleus, and function as ligand-dependent transcription factors. That is to say, the hormone-receptor complex binds to promoter regions of responsive genes and stimulates or sometimes inhibit transcription from those genes.

Thus, the mechanism of action of steroid hormones is to modulate gene expression in target cells. By selectively affecting transcription from a battery of genes, the concentration of those respective proteins are altered, which clearly can change the phenotype of the cell (Bowen, 1998).

Structure of Intracellular Receptors

Steroid and thyroid hormone receptors are members of a large group ("superfamily") of transcription factors. In some cases, multiple forms of a given receptor are expressed in cells, adding to the complexity of the response. All of these receptors are composed of a single polypeptide chain that has, in the simplest analysis, three distinct domains:

- The amino-terminus: In most cases, this region is involved in activating or stimulating transcription by interacting with other components of the transcriptional machinery. The sequence is highly variable among different receptors.

- DNA binding domain: Amino acids in this region are responsible for binding of the receptor to specific sequences of DNA.

- The carboxy-terminus or ligand-binding domain: This is the region that binds hormone.

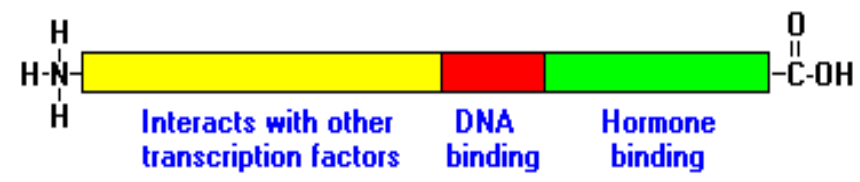

In addition to these three core domains, two other important regions of the receptor protein are a nuclear localization sequence, which targets the protein to nucleus, and a dimerization domain, which is responsible for latching two receptors together in a form capable of binding DNA (Wikipedia, 2014).

Hormone-Receptor Binding and Interactions with DNA

Being lipids, steroid hormones enter the cell by simple diffusion across the plasma membrane. Thyroid hormones enter the cell by facilitated diffusion. The receptors exist either in the cytoplasm or nucleus, which is where they meet the hormone. When hormone binds to receptor, a characteristic series of events occurs:

- Receptor activation is the term used to describe conformational changes in the receptor induced by binding hormone. The major consequence of activation is that the receptor becomes competent to bind DNA.

- Activated receptors bind to "hormone response elements", which are short specific sequences of DNA which are located in promoters of hormone-responsive genes. In most cases, hormone-receptor complexes bind DNA in pairs, as shown in the figure below.

- Transcription from those genes to which the receptor is bound is affected. Most commonly, receptor binding stimulates transcription. The hormone-receptor complex thus functions as a transcription factor (Schwabe, et al 1993).

As might be expected, there are a number of variations on the themes described above, depending on the specific receptor in question. For example, in the absence of hormone, some intracellular receptors do bind their hormone response 
elements loosely and silence transcription, but, when complexed to hormone, become activated and strongly stimulate transcription. Some receptors bind DNA not with another of their kind, but with different intracellular receptor (Bowen, 1998).

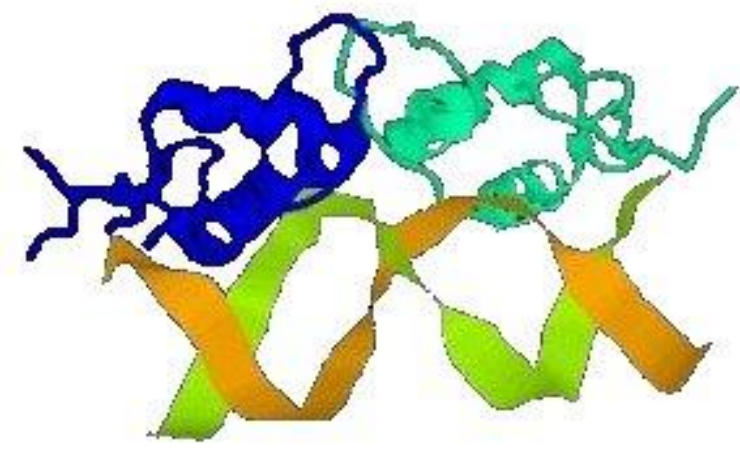

As a specific example, consider glucocorticoid, a type of steroid hormone that probably affects the physiology of all cells in the body. The image to the right depicts a pair of glucocorticoid receptors (blue and green on the top) bound to their DNA hormone response element (bottom). The two steroid hormones are not visible in this depiction.

The consensus sequence of the hormone response element in this case (called a glucocorticoid response element) is GGTACANNNTGTTCT, where $\mathrm{N}$ is any nucleotide (Evans, 1988).

Nuclear receptors: Steroid receptors of the nuclear receptor family are all transcription factors. Depending upon the type of receptor, they are either located in the cytosol and move to the cell nucleus upon activation, or remain in the nucleus waiting for the steroid hormone to enter and activate them. This uptake into the nucleus is facilitated by nuclear localization signal (NLS) found in the hinge region of the receptor. This region of the receptor is covered up by heat shock proteins (HSPs) which bind the receptor until the hormone is present. Upon binding by the hormone the receptor undergoes a conformational change releasing the HSP and the receptor together with the bound hormone enter the nucleus to act upon transcription.

This list is incomplete. It does not include the seco-steroid calcitriol (vitamin D)

- Nuclear receptors

- Subfamily 3: Estrogen Receptor-like

- Group A: Estrogen receptor (Sex hormones: Estrogen)

- 1: Estrogen receptor- $\alpha$ (ER $\alpha$; NR3A1, ESR1)

- 2: Estrogen receptor- $\beta$ (ER $\beta$; NR3A2, ESR2)

- Group B: Estrogen related receptor

- 1: Estrogen-related receptor- $\alpha$ (ERR $\alpha$; NR3B1, ESRRA)

- 2: Estrogen-related receptor- $\beta$ (ERR $\beta ;$ NR3B2, ESRRB)

- 3: Estrogen-related receptor- $\gamma$ (ERR $\gamma ;$ NR3B3, ESRRG)

- Group C: 3-Ketosteroid receptors

- 1: Glucocorticoid receptor (GR; NR3C1) (Cortisol)

- 2: Mineralocorticoid receptor (MR; NR3C2) (Aldosterone)

- 3: Progesterone receptor (PR; NR3C3, PGR) (Sex hormones: Progesterone)

- 4: Androgen receptor (AR; NR3C4, AR) [Sex hormones: Testosterone] (Schwabe, et al 1993).

\section{SOME CHARACTERISTICS OF SIGNAL CONDUCTION}

\section{A. Signal Amplification (Spare receptors)}

Many receptors, particularly those that respond to hormones, neurotransmitters, and peptides, posses the ability to amplify signal duration and intensity. The family of $G$ protein-linked receptors exemplifies many of the possible responses initiated by ligand binding to a receptor. Specifically, two phenomena account for the amplification of the ligand-receptor signal (Michelle, et al 2012).

First, a single ligand-receptor complex can interact with many $G$ proteins, thereby multiplying the original signal many-fold.

Second, the activated $G$ proteins persist for a longer duration than the original ligand-receptor complex. The binding of albuterol, for example, may only exist for a few milliseconds, but the subsequent activated $G$ proteins may last for hundreds of milliseconds.

Spare receptors are exhibited by insulin receptors, where it has been estimated that 99 percent of the receptors are "spare." This constitutes an immense functional reserve that ensures adequate amounts of glucose enter the cell. On the other end of the scale is the human heart, in which about five to ten percent of the total $\beta$-adrenoceptors are spare. An important implication of this observation is that little functional reserve exists in the failing heart; most receptors must be occupied to obtain maximum contractility (Michelle, et al 2012).

\section{B. Desensitisation, Downregulation and upregulation}

Down-regulation is the process by which a cell decreases the quantity of a cellular component, such as RNA or protein, in response to an external variable. An increase of a cellular component is called upregulation.

An example of downregulation is the cellular decrease in the number of receptors to a molecule, such as a hormone or neurotransmitter, which reduces the cell's sensitivity to the molecule. This phenomenon is an example of a locally acting negative feedback mechanism (Sherwood, 2004).

An example of upregulation is the increased number of cytochrome P450 enzymes in liver cells when xenobiotic molecules such as dioxin are administered (resulting in greater degradation of these molecules).

Some receptor agonists may cause down-regulation of their respective receptor(s), while most receptor antagonists may temporally upregulate their respective receptor(s). The disequilibrium caused by these changes often causes withdrawal when the long-term use of a medication or drug is discontinued. However, the use of certain receptor antagonists may also damage receptors faster than they upregulate (internalization of receptors due to antagonism) Wilmore \& Costill, 2004.

Upregulation and down-regulation can also happen as a response to toxins or hormones. An example of upregulation in pregnancy is hormones that cause cells in the uterus to become more sensitive to oxytocin.

Repeated or continuous administration of an agonist (or an 
antagonist) may lead to changes in the responsiveness of the receptor. To prevent potential damage to the cell (for example, high concentrations of calcium, initiating cell death), several mechanisms have evolved to protect a cell from excessive stimulation. When repeated administration of a drug results in a diminished effect, the phenomenon is called tachyphylaxis. The receptor becomes desensitized to the action of the drug. In this phenomenon, the receptors are still present on the cell surface but are unresponsive to the ligand (Michelle, et al 2012).

\section{DOSE-RESPONSE RELATIONSHIPS}

An agonist is defined as an agent that can bind to a receptor and elicit a biologic response. The magnitude of the drug effect depends on the drug concentration at the receptor site, which in turn is determined by the dose of drug administered and by factors characteristic of the drug pharmacokinetic profile, such as rate of absorption, distribution, and metabolism.

\section{A. Graded dose-response relations}

As the concentration of a drug increases, the magnitude of its pharmacologic effect also increases. The relationship between dose and response is a continuous one, and it can be mathematically described for many systems by application of the law of mass action, assuming the simplest model of drug binding:

The response is a graded effect, meaning that the response is continuous and gradual.

This contrasts with a quantal response, which describes an all-or-nothing response. A graph of this relationship is known as a graded dose-response curve. Plotting the magnitude of the response against increasing doses of a drug produces a graph that has the general shape of a rectangular hyperbola - a very familiar curve in biology, because it can be applied to diverse biological events, such as ligand binding, enzymatic activity, and responses to pharmacologic agents.

- Potency: Two important properties of drugs can be determined by graded dose-response curves.

The first is potency, a measure of the amount of drug necessary to produce an effect of a given magnitude. For a number of reasons, the concentration producing an effect that is fifty percent of the maximum is used to determine potency; it commonly designated as the $\mathrm{EC}_{50}$. The $\mathrm{EC}_{50}$ for Drugs $\mathrm{A}$ and $\mathrm{B}$ can be indicated. If drug $\mathrm{A}$ is more potent than Drug $\mathrm{B}$ it may mean that less Drug $A$ is needed to obtain 50 percent effect. Thus, therapeutic preparations of drugs will reflect the potency. For example, candesartan and irbesartan are angiotensin-receptor blockers that are used alone or in combination to treat hypertension. Candesartan is more potent than irbesartan because the dose range for candesartan is 4 to $32 \mathrm{mg}$, as compared to a dose range of 75 to $300 \mathrm{mg}$ for irbesartan. Candesartan would be Drug A and irbesartan would be Drug B.

Efficacy [intrinsic activity]: The second drug property that can be determined from graded dose-response plots is the efficacy of the drug. This is the ability of a drug to illicit a physiologic response when it interacts with a receptor. Efficacy is dependent on the number of drug-receptor complexes formed and the efficiency of the coupling of receptor activation to cellular responses.

\section{REFERENCES}

[1] Aaftaab Sethi, Khusbhoo Joshi, K. Sasikala and Mallika Alvala. Molecular Docking in Modern Drug Discovery: Principles and Recent Applications. Inteck Open. July 2nd 2019. doi: 10.5772/intechopen.85991.

[2] Alberts, B. (2008). "Signaling through enzyme linked receptors" in Molecular Biology of the Cell, 5th Edition. New York, Garland Science

[3] Amrita Virtual Lab. Collaborative Platform. (20014). [Ver. 00.3.2].

[4] Angers, S, Salahpour, A, Bouvier, M. (2002). Dimerization: an emerging concept for $\mathrm{G}$ protein-coupled receptor ontogeny and function. Annu Rev Pharmacol Toxicol. 42:409-35.

[5] Ashcroft, F. M., Ed. Ion Channels and Disease; Academic Press: San Diego, CA, 2000.

[6] Barnard, E. Trends Biochem. Sci., 1992, 17, 368-374.

[7] Bertil, H. (2009). G Protein-Coupled Receptor. University of Washington School of Medicine, Seattle, WA Scholarpedia, 4(12):8214.

[8] bio.libretexts.org>...>8: signaling 2019. 8.4: Gprotein Coupled Receptorss (GPCR) Bio Libre Texts

[9] Bowen, R. (1998). Mechanism of Action: Hormones with Intracellular Receptors. rbowen@1amar.colostate.edu. Last updated May 27, 1998.

[10] Christopoulos, A,\& El-Fakahany, EE. (1999). "Qualitative and quantitative assessment of relative agonist efficacy". Biochem. Pharmacol. 58 (5): 735-48. doi:10.1016/S0006-2952(99)00087-8. PMID 10449182.

[11] Diego Guidolin, Manuela Marcoli, Cinzia Tortorella, Guido Maura, Luigi F. Agnati. Receptor-Receptor Interactions as a Widespread Phenomenon: Novel Targets for Drug Development? Frontiers in Endocrinology (Lausanne). doi: 10.3389/fendo. 2019.00053 PMCID: PMC6387912. PMID: 30833931

[12] Evans, RM. (1988). "The steroid and thyroid hormone receptor superfamily". Science $240 \quad$ (4854): 889-95. 마: 10.1126/science.3283939. PMID 3283939.

[13] Heyes, J. (2014). Enzyme-linked Receptors: Manchester University,

[14] http://www.youtube.com/watch?v=QoJBIfM0bmU http://www.wiley.com/college/fob/quiz/quiz21/21-15.html nd \& http://www.youtube.com/watch?v=ZZ1uu0rXFDc\&feature $=$ related nd.

[15] http://www. www.wikipedia,com.

[16] Iain, LOB. (2006). Pharmacokinetics and Pharmacodynamics. The Dynamics of Drug Absorption, Distribution and Elimination. In Goodman \& Gilman's The Pharmacological Basis of Therapeutics$11^{\text {th }}$ Ed. (2006). McGraw-Hill. New York.

[17] Juan Zhao, YulinDeng , Zhaotan Jiang, Hong Quing. G Protein-Coupled Receptors (GPCRs) in Alzheimer's Disease: A Focus on BACE1 Related GPCRs. Aging Neurosci., 24 March 2016 https://doi.org/10.3389/fnagi.2016.00058

[18] Kenakin, T. (2004) Principles: Receptor theory in pharmacology Trends Pharmacol Sci Vol 25 No.4

[19] Kenakin, T. (2008). "What systems can and can't do". Br. J. Pharmacol. 153 (5): 841-3. $\underline{\text { doi: } 10.1038 / \text { sj.bjp.0707677. }}$ PMC 2267279. PMID 18204481.

[20] Lodish, H. (2008). "Cell Signaling I and II" in Molecular Cell Biology, 6th Edition. New York, W. H. Freeman and Company.

[21] Meenakshi Gupta, Ruchika Sharma, Anoop Kumar. Docking Techniques in Pharmacology: How Much Promising? Comput Biol Chem. 2018 Oct;76:210-217. PMID: 30067954. doi: 10.1016/j.compbiolchem.2018.06.005. Epub 2018 Jul 3.

[22] Michelle, AC, Richard, F, Jose, AR, Karen, W, Richard, AH. (2012) Lippincott Illustrated Reviews ( $5^{\text {th }}$ Edition): Pharmacology. Unit 1 , Introduction to Pharmacology; Chapter 2: Drug-Receptor Interactions and Pharmacodynamics. Wolters Kluwer / Lippincott Williams \& Wilkins Health. International Edition; 2012.

[23] Naveen Chhabra, Madan L Aseri, Deepak Padmanabhan. A Review of Drug Isomerism and its Significance. International Journal of Applied Basic Medical Research. Int J Appl Basic Med Res 2013 Jan-Jun; 3(1): 16-18. doi: 10.4103/2229-516X.11223. Int J Appl Basic Med Res. v.3(1); Jan-Jun 2013 PMC3678675.

[24] Rang, HP. (2006). "The receptor concept: pharmacology's big idea". Br. J. Pharmacol. 147 (S1): S9-16. doi:10.1038/sj.bjp.0706457. PMC 1760743. PMID 16402126.

[25] Rasmussen, SG, DeVree, BT, Zou, Y, Kruse, AC, Chung, KY, Kobilka, TS, Thian, FS, Chae, PS, Pardon, E, Calinski, D, Mathiesen, JM, Shah, ST, Lyons, JA, Caffrey, M, Gellman, SH, Steyaert, J, Skiniotis, G, Weis, WI, Sunahara, RK, Kobilka, BK. (2011). Crystal structure of the $\beta 2$ adrenergic receptor-Gs protein complex. Nature. 477(7366):549-555. doi: 10.1038/nature10361. 
[26] Ross, EM \& Kenakin, TP. (2001). Pharmacodynamics. Mechanisms of drug action and the relationship between drug concentration and effect. In Goodman \& Gilman's The Pharmacological Basis of Therapeutics, Vol. Tenth. J.G. Hardman \& L.E. Limbird, Eds. McGraw-Hill. New York.

[27] Schwabe, JW, Chapman, L, Finch, JT, Rhodes, D. (1993). "The crystal structure of the estrogen receptor DNA-binding domain bound to DNA: how receptors discriminate between their response elements". Cell 75 (3): 567-78. Doi: 10.1016/0092-8674(93)90390-C. PMID 8221895 .

[28] Sherwood, L. (2004). "Human Physiology From Cells to Systems, 5th Ed" (p. 680). Belmont, CA: Brooks/Cole-Thomson Learning

[29] Shupeng Li, Albert HCW, Fang Liu. Ligand-Gated Ion Channel Interacting Proteins and Their Role in Neuroprotection. Frontiers In Cellular Neuroscience. Front. Cell. Neurosci., 09 May 2014 | https://doi.org/10.3389/fncel.2014.00125.

[30] Stephenson, RP. (1997). A Modification of Receptor Theory: Br J Pharmacol. Feb 1997; 120(Suppl 1): 106-120. Doi: 10.1111/j.1476-5381.1997.tb06784.x PMCID: PMC3224279.

[31] Tanner Marshall, MS, Evan Debevec-McKenney, Antonia Syrnioti, Yifan Xiao M.D. osmosis. Pharmacodynamics: Drug-receptor $\begin{array}{llll}\text { interactions. } & \text { Mploaded } & \text { Mar } & \end{array}$ www.osmosis.org>learn.pharmacodynamics_drugs-rec...

[32] Venkatakrishnan, AJ, Deupi, X, Lebon, G, Tate, CG, Schertler, GF, Babu, MM. (2013). Molecular signatures of G-protein-coupled receptors. Nature. 494(7436):185-94. doi: 10.1038/nature11896.

[33] Wilmore, J. Costill, D. (2004). Physiology of Sport and Exercise, 3rd $E d$ (p. 164). Champaign, IL: Human Kinetics.

[34] www.sciencedirect.com>topics.ligand-gated-ion-channel.

[35] www.ncbi.nih.gov>books>NBK26822. 